\title{
My Continuing Journey from Logic to Computational Argumentation
}

\author{
Douglas Walton ${ }^{1}$ (1)
}

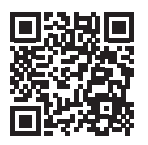

${ }^{1}$ Prof. Dr., Institution, Faculty and/or Department,

City-Country, Centre for Research in Reasoning, Argumentation and Rhetoric, University of Windsor, Canada

ORCID: D.W. 0000-0003-0728-1370

Sorumlu yazar/Corresponding author: Douglas Walton,

Centre for Research in

Reasoning, Argumentation and Rhetoric, University of Windsor, Canada

E-mail/E-posta: waltoncrrar@gmail.com

Başvuru/Submitted: 23.11.2019

Kabul/Accepted: 02.12 .2019

\section{Atıf/Citation:}

Walton, Douglas. (2019). “My Continuing Journey from Logic to Computational Argumentation" Felsefe Arkivi-Archives of Philosophy, 51: 321-330.

https://doi.org/10.26650/arcp2019-5123

\section{ABSTRACT}

This paper begins with a brief account of how I started out as a young logician studying modal logic with the hope that it would be useful when applied to evaluating real examples of arguments found in natural language texts. The exposition moves on to relate how my interests shifted to the study of argumentation in informal logic, and from there to computational systems combining defeasible argumentation schemes with argument mapping (diagramming). The story ends by leading to recent collaborations with three young logicians and computer scientists who are continuing on the path of my journey.

Keywords: Argumentation, informal logic, history of logic, future of logic

\section{Introduction}

This paper begins in section 2 with a very brief account of how I started out as a young logician studying modal logic and was corrupted from this pure abstract pursuit by falling in with informal logicians, argumentation scholars and computer scientists who wanted to make logic into a partly practical discipline. My motive was the hope that abstract normative models of reasoning could be useful when applied to practical tasks of interpreting, identifying, analyzing 
and evaluating real examples found in natural language texts. Section 3 indicates how the study of argumentation theory of this sort was taken up by computer scientists. One tool central to the computational systems approach is briefly described and explained in section 4, the defeasible argumentation schemes. This section briefly explains, by presenting an example, how argumentation schemes have been combined with argument mapping, sometimes also called argument diagramming in logic. Section 5 explains how my lifetime project of pursuing this line of research continues through recent collaborations with three young logicians and computer scientists who share my research goals and methods.

\section{From Modal Logic to Argumentation}

The dominant model for research when I was a young logician was deductive logic. In 1975 I spent a sabbatical year at Victoria University of Wellington in New Zealand studying modal logic with George Hughes and Max Cresswell. ${ }^{1}$ However, I had a problem trying to convince my logic students to figure out how possible worlds could be applied with satisfying results to real argumentation of the kind they encountered in their occupations and daily lives. While I was in New Zealand (1975), my wife and I went for a visit to Australia. While giving an invited lecture at the University of New South Wales in Sydney, I had the good fortune to spend part of one day with Charles Leonard Hamblin. This visit turned out to be very valuable to me, because just a few years before I had taken up the study of informal fallacies, a field which I thought had been sadly under-investigated at the time (and still is) even though it appeared to be very useful from a practical point of view.

Hamblin's book (1970) took a radically different dialectical approach to fallacies and to practical logic generally by building and applying formal dialogue models that he called dialectical systems. At that time his approach increasingly began to impress me as potentially very promising, because as a logic teacher I had also begun to take up the study of argumentation and informal logic in a much more dedicated way. Informal logic appealed to me as a good direction because of its potential practical usefulness for analyzing everyday examples of arguments commonly found in natural language discourse.

Hamblin $(1970,256)$ wrote that dialectical systems can be studied either normatively, meaning that they are meant to provide rules and procedures to model correct argumentation, or descriptively, meaning that they are meant to be applied to real examples of natural language texts. In his view, both sides of logic, the formal side and the applied side, are required: "Neither approach is of any importance on its own; for descriptions of actual cases must aim to bring out formalizable features, and formal systems must aim to throw light on actual, describable phenomena." Since that time, many formal and computational argumentation systems in artificial intelligence have followed Hamblin's route in insisting on the use of collecting real examples from natural language discourse.

The next big shift in the direction of my personal area of study was the collaborative work with argumentation theorists who I had met in Europe during two separate years of collaborative

1 https://en.wikipedia.org/wiki/Modal_logic 
work at NIAS, the Netherlands Institute for Advanced Study². During the first year I worked with Erik Krabbe on Hamblin-style dialogue theory, resulting in the book (Walton and Krabbe, 1995). During a second visit to NIAS, I worked with a group of investigators led by Frans van Eemeren and Rob Grootendorst (Van Eemeren and Grootendorst, 2004). After that time computer scientists began to take a serious interest in argumentation as an area of application for them, especially in artificial intelligence. This initiative to combine argumentation with artificial intelligence was undertaken by eleven researchers from argumentation theory and eleven computer scientists who met in the Highlands of Scotland in an isolated castle near Pitlochry for one week to produce a book together (Reed and Norman, 2004). My direction of research from that point to the present was strongly influenced by this turn of events. Nowadays, there are productive collaborations between argumentation theorists and computer scientists, most notably in the fields of artificial intelligence and multiagent systems. This multidisciplinary synergy was, as I can see in retrospect, a natural outcome of the pioneering work done by Charles Hamblin, a logician who had made serious contributions to computer science as well. ${ }^{3}$

\section{Formalizing Informal Logic with Computers}

The Carneades Argumentation System formalizes argumentation by using bipartite directed graphs of the kind typically called argument diagrams in informal logic, consisting of single arguments, linked arguments, convergent arguments, and other types of arguments explained below by using the example in figure 1. Carneades can be used for argument construction (often called argument invention in rhetoric) as well as argument reconstruction. In argument reconstruction of the kind familiar in informal logic, a particular text in a natural language is interpreted and analysed by building an argument diagram. Argument diagrams (or maps) visualize the arguments found in natural language sources using argument graphs. Arguments can be visualized in different ways and at levels of abstraction, for different purposes.

Formally, an argument graph is a 4-tuple $\langle S, A, P, C\rangle$, where $S$ is a set of statement nodes, $A$ is a set of argument nodes, $P$ is a set of premises, and $C$ is a set of conclusions (Gordon, 2010). The 4-tuple does not model a single argument, but rather a set of arguments, a whole argument graph. A single argument, a linked argument, a convergent argument or a sequence of chained arguments, can function as subgraphs of a larger, more comprehensive argument graph.

To see an example, look ahead to figure 1 . The statement nodes are shown as the rectangular text boxes in the figure containing statements. The argument nodes are the circles containing plus or minus signs. Argument graphs are evaluated, relative to what are called audiences in the argumentation literature, to determine the acceptability of statements in a stage of dialogue (Gordon and Walton, 2009). Each dialogue has an opening stage, an argumentation stage and a closing stage. Audiences are modelled as a set of assumptions and an assignment of weights to argument nodes. Where $L$ is a propositional language as defined in the formal Carneades

2 https://nias.knaw.nl/

3 https://en.wikipedia.org/wiki/Charles_Leonard_Hamblin 
system, an audience is a structure <assumptions, weight $>$, where assumptions $\subseteq L$ is a consistent set of propositions assumed to be accepted by or acceptable to the audience and weight is a partial function mapping arguments to real numbers in the range $0.0 \ldots 1.0$, representing the relative weights (stronger or weaker) assigned by the audience (Gordon and Walton, 2011).

Conflicts between pro and con arguments are resolved using proof standards (Gordon and Walton, 2009) that determine how much proof is required for the statement to be taken as acceptable (presumably true). The proof standard is used in the formal model to determine the acceptability of the statements and arguments that are parts of the argument graph yielded by interpreting and analyzing the text of discourse quoted as representing the given argument that is to be identified, analysed and evaluated.

\section{Argumentation Schemes in Formal and Computational Systems}

Carneades also formalizes many of the argumentation schemes used to construct or reconstruct arguments, as well as to check whether arguments are "valid", i.e. whether they properly instantiate the types of argument fitting particular arguments in the example to be analysed. Here we can use the example of the scheme for argument from expert opinion to illustrate how this works. When you apply the computational system to a real example you can access the list of the argumentation schemes available and choose which one to apply.

For example here is the standard version of the scheme for argument from expert opinion that can be found in (Walton, Reed and Macagno, 2008, 217).

Major Premise: Source $E$ is an expert in subject domain $D$ containing proposition $A$.

Minor Premise: $E$ asserts that proposition $A$ is true (false).

Second Minor Premise: $A$ is within $D$.

Conclusion: A may be presumed to be true (false).

It can be added that there are different versions, ways of formalizing the scheme for different purposes, but each version can be shown to be structurally equivalent to the others. One is to break the standard scheme into three separate premises, one stating that $E$ is an expert, a second one specifying the subject domain of the area of expertise, and a third one stating that the domain $D$ contains the proposition $A$. Another way is to use the following heuristic form of the scheme: an expert says that $A$ is true therefore $A$ is tentatively acceptable as true (subject to the asking of critical questions indicating that $A$ might not be true). The heuristic version is very helpful for teaching students how to grasp the essence of the scheme in a simple way, so that they can learn to identify it as a common form of argument that makes sense to them. But a weakness of the heuristic version is that an uncritical arguer may be tempted to jump to the conclusion without being aware of qualifications expressed by the critical questions that also need to be considered. According to another way, a conditional premise can be added as a general rule (defeasible generalization) such as: if $E$ is an expert in $S$ containing $A$ and $E$ asserts that $A$ is 
true (false), then $A$ is true (false). When the scheme is interpreted in this way, the argument from expert opinion takes the form called defeasible modus ponens: if $A$ then defeasibly $B, A$; therefore $B$ is tentatively acceptable as a presumption.

These are the six basic critical questions matching the scheme for argument from an expert (Walton, 2008, 218; Walton, Reed and Macagno, 2008, 310):

Expertise Question: How credible is $E$ as an expert source?

Field Question: Is $E$ an expert in the domain $S$ that $A$ is in?

Opinion Question: What did $E$ assert that implies $A$ ?

Trustworthiness Question: Is E personally reliable as a source?

Consistency Question: Is $A$ consistent with what other experts assert?

Backup Evidence Question: Is Es assertion based on evidence?

The set of critical questions can be used used to pinpoint the weak points in the given argument and suggest counter arguments, for example to a student learning informal logic who may have difficulties figuring out how to respond to a given argument.

When an argument analyst asks any one of the six critical questions, the original argument is tentatively suspended until the critical question has been responded to adequately. Once the question has been answered however, the argument from expert opinion is restored to its original status as a plausible argument. Thus a burden of proof can shift back and forth as critical questions are asked and answered. If no scheme matches the argument in the given text you are trying to reconstruct, you can put in no scheme, or classify the type of argument under a more general scheme such as modus ponens, either in its familiar deductive form or as a defeasible form of argument that shifts a weight of presumption from the premises of an argument to its conclusion. In Carneades, the critical questions matching a scheme are represented as additional premises formalized as either assumptions or exceptions in the formal model represented in the computational system in all four of its versions.

The top 25 of Walton's argumentation schemes (Gordon, Friedrich and Walton, 2018) are available on Github as programmed in YAML ${ }^{4}$, and the remaining 81 argumentation schemes are listed and classified in classified in the system of Walton, Reed and Macagno (2008). This programming was conducted within the system of the master's thesis entitled Douglas Walton's Argumentation Schemes and Applications prepared by Başak Kurtuldu under the supervision of Dr. Vedat Kamer, in the Department of Philosophy, Istanbul University. ${ }^{5}$ This means that a grand total of 106 argumentation schemes have been implemented for the Carneades System. More schemes are currently being identified that can be added to this list.

4 https://github.com/carneades/carneades-4/blob/master/examples/AGs/YAML/walton.yml

5 https://github.com/basakkurtuldu/walton-argumentation-schemes-for-carneades-4 


\section{Combining Schemes with Diagrams: an Example}

Schemes can be combined with argument diagrams in a way that can intuitively be made clear by using version 2 of the system. Carneades went progressively through four versions. The source codes for all four versions can be accessed on the Internet. ${ }^{6}$ All four versions model arguments as directed graphs. Version 2, which will be used in example 1 to illustrate some basic features, is nicely displayed in this example that makes the basics of the technology easy to grasp. Version 4, the current version, is the most technically advanced, but all the versions share the core concepts that are needed to get a basic grasp of how the argumentation system works when applied to examples of the kinds of tasks typically emphasized in informal logic and argumentation studies generally.

Premises and conclusions are visualized as lines and arrows, respectively, connecting statement and argument nodes. For an example the reader can look at figure 1, which represents three arguments and a conclusion. This example argument is drawn by the Carneades system, once the user has input the structure of the argumentation, including the ultimate proposition to be proved, and indicated which of the propositions acting as premises are accepted, rejected, or neither accepted nor rejected. The third category is represented by a rectangle with no background, in other words a white rectangle, but there are no instances of this in this particular example.

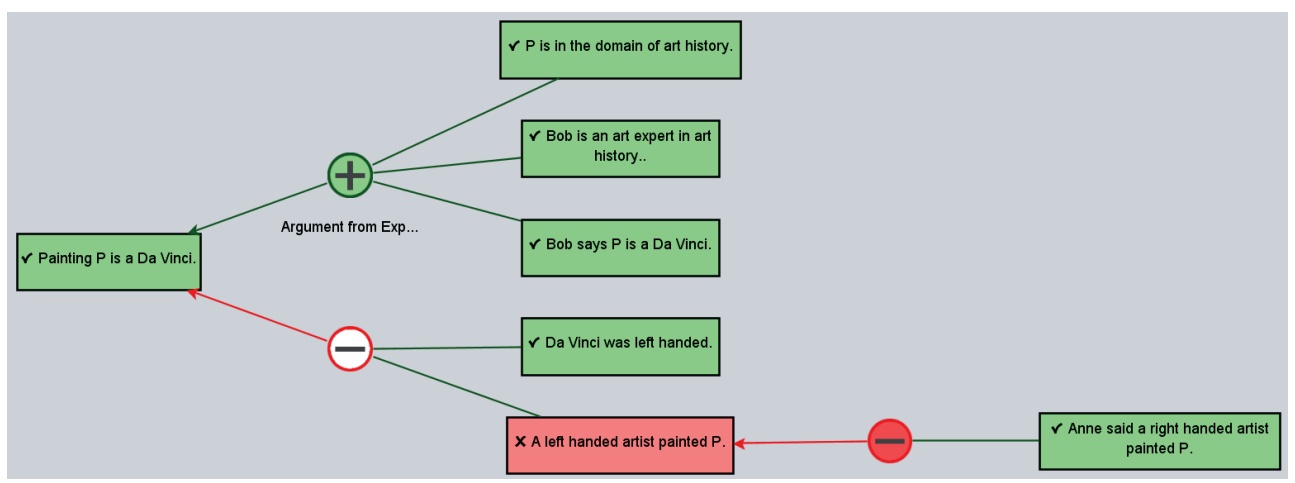

Figure 1: A Carneades Argument Map of an Example Argument

You can see how a typical argument of the kind studied in informal logic is modeled by taking a look at the argument map shown in figure 1. The sequence of argumentation flows from right to left. Hence the proposition appearing in the leftmost rectangle, the statement that painting $\mathrm{P}$ is a $\mathrm{Da}$ Vinci, is the ultimate conclusion to be proved or disproved. The pro argument at the top, as shown by the notation under its argument node, is an instance of the argumentation scheme for argument from expert opinion. This argument is linked, according to the kinds of argument structures recognized in informal logic. In a linked argument all the premises go together to

6 https://github.com/carneades 
support the conclusion. Since all three premises in this argument are shown as accepted, indicated by the three rectangles shown with a green background, the conclusion, stating that painting $\mathrm{P}$ is a Da Vinci, is also shown as accepted.

Next we have to compare the pro argument at the top with the con argument at the bottom. Its being a con argument is indicated by the minus sign in its argument node. This argument has two premises. One is the premise that Da Vinci was left-handed, which we will take as accepted based on some factual evidence not shown in the diagram. The other premise is the statement shown in the red coloured box, claiming that a left-handed artist painted $\mathrm{P}$. These two premises form a linked argument, so if both premises were to be accepted, the argument would support the ultimate conclusion. Presumably there needs to be some sort of implicit premise to the effect that not many people are left-handed. However, we will ignore implicit premises based on common background knowledge to keep the example simple.

Next, notice that at the bottom right, there is another con argument based on the premise that someone called Anne said that a right-handed artist painted P. This statement attacks the proposition shown to the left of it stating that a left-handed artist painted P. Because this latter proposition is rejected, as shown by its being presented in a rectangle with a red background, the linked argument of which it is part fails to refute the ultimate conclusion that painting $\mathrm{P}$ is a $\mathrm{Da}$ Vinci. Therefore, even though the ultimate conclusion is attacked by a con argument, it is not strong enough to refute the pro argument from argument from expert opinion. Therefore once all the information from the text of the example is put into the Carneades Argumentation System in the manner indicated by figure 1 , what is shown is that the ultimate conclusion is supported by the sequence of argumentation strongly enough to meet the standard of proof appropriate for the argumentation in this example. The standard of proof has to be input by the user. Such standards can include the preponderance of the evidence standard, the beyond reasonable doubt standard, and so forth. Different standards need to be used depending on the context of the argumentation in the example, which is modelled by the type of dialogue fitting the context. A type of dialogue is formally modelled by its protocols, including its standard of proof and the kinds of speech acts allowed as moves in the formal model.

The interesting thing about this example is that it shows how arguments of this sort typically studied in informal logic, comprise single arguments, multi-premised arguments of the two types, linked arguments and convergent arguments, as well as more complex sequences of argumentation of the kind identified in computer sciences as chaining of argumentation. In a chained argument, the conclusion of one argument becomes a premise in the second argument resulting in a sequence of argumentation that can be carried forward recursively.

What the examples suggest is that typical arguments of the type studied in informal logic can be formally modelled using a formal and computational argumentation system such as Carneades. However, it also needs to be noted that this formalism is currently incomplete with regard to its purpose of formalizing informal logic for two reasons. This system does not model 
relevance of argumentation. And at least insofar as the system is explained here, it does not cumulative argumentation (Walton and Gordon, 2017), the kind of chained argumentation where there is a buildup of evidence through various steps in the sequence of argumentation making this chain of arguments stronger and stronger with regard to the support it gives to the ultimate conclusion.

\section{Young Logicians and Computer Scientists}

In more recent years I have enjoyed working with younger people and reading their work and I might mention three. Dr. Marcin Koszowy is an Assistant Professor in the Department of Logic, Cognitive Science and Philosophy of Science, Institute of Philosophy, University of Białystok, Poland. He is also a postdoctoral researcher in the Institute of Philosophy and Sociology of the Polish Academy of Sciences, Warsaw, Poland, and a Member of the Computational Ethos Lab (CELab) in the Centre for Argument Technology (ARG-tech). ${ }^{7}$ One of our joint projects was to lay foundations for disentangling arguments from deontic authority and arguments from expert authority by applying the Carneades Argumentation System to period.

An objective of our work has been to show how the distinction between arguments from expert opinion and deontic authority plays an explicatory role in identifying different kinds of instances of the argumentum ad verecundiam fallacy. To pursue this goal we focused on the ad verecundiam fallacy by employing the profiles of dialogue method (Krabbe 1999). Using this method we identified a species of the fallacy that works by forestalling of questioning in arguments from expert opinion (Koszowy and Walton, 2017). A profile of dialogue is a graph structure used to model a sequence of speech acts surrounding both the putting forward of an argument and the responses to it at the next moves in a dialogue. This method was applied to a instance of cross-examining a software engineer in a legal deposition regarding a case of intellectual property litigation.

For the past seven years or so I have been working with Dr. Alice Toniolo, a lecturer in the School of Computer Science at the University of St. Andrews. ${ }^{8}$ Her research is in the area of multiagent systems currently being applied to facilitate human reasoning and decision-making under uncertainty. She and I are both interested in models of argumentation-based reasoning and dialogue to handle conflicting and uncertain information. Her recent research has been on the topics of normative reasoning, planning, deliberation, evidential reasoning, provenance and intelligence analysis. We have published articles on formal models of deliberation dialogue. A recent example of this work (Walton, Toniolo and Norman, 2019), carried out with Professor Tim Norman of the University of Southampton, builds a typology of deliberation dialogues by presenting a series of realistic examples of deliberation in the tradition of Hamblin $(1970,256)$. The observations from our examples are used to suggest that in order to better understand and contextualise the argumentation components that make up realistic deliberations, it is necessary to carefully distinguish between two species of deliberation called problem solving deliberation and decision making deliberation.

7 For some of his papers see: https://www.researchgate.net/profile/Marcin_Koszowy

8 For some of her recent papers see: https://www.cs.st-andrews.ac.uk/directory/person?id=at258 
A young computer scientist from Brazil, Dr. Alison R. Panisson, has applied argumentation tools to widely interesting problems and issues that arise not only in computer science, but are also logical in nature and that have important applications to argumentation studies. ${ }^{9}$ I met him at a meeting of the COMMA conference in 2018. His current research interests are in artificial intelligence, multiagent systems, semantic technologies, argumentation, and theory of mind. On his website, he writes "I am interested in applying argumentation techniques to develop explainable AI". He is currently a Research fellow at PUCRS (Pontifícia Universidade Católica do Rio Grande do Sul) where he did his PhD work in Computer Science (2015- 2019). His current research project concerns applications of argumentation techniques in artificial intelligence. He has recently written papers on several topics that are of central interest to argumentation theorists; formal semantics of speech acts for argumentative dialogues, choosing appropriate arguments from trustworthy sources, practical argumentation-based dialogues in multi-agent systems, and formal semantics of theory of mind in agent communication. For an example see Panisson et al. (2018).

Conflict of Interest: The authors declare that they have no conflicts of interest

\section{References}

Gordon, T. F. (2010). The Carneades Argumentation Support System, in Dialectics, Dialogue and Argumentation, C. Reed and C. W. Tindale (eds.). London: College Publications, 145-156.

Gordon, T. F. \& Walton, D. (2009). Proof Burdens and Standards, in Argumentation in Artificial Intelligence, I. Rahwan and G. Simari (eds.). Dordrecht: Springer, 239-258.

Gordon, T. F., Friedrich, H. and Walton, D. (2018).Representing Argumentation Schemes with Constraint Handling Rules, Argument \& Computation, 9(2), 91-119.

Hamblin, C. L. (1970). Fallacies. London: Methuen.

Koszowy, M. and Walton, D. (2017). Profiles of Dialogue for Repairing Faults in Arguments from Expert Opinion, Logic and Logical Philosophy, 26, 1, 79-113.

Krabbe, E. C. W. (1999). Profiles of Dialogue. In J. Gerbrandy, M. Marx, M. de Rijke, \& Y. Venema (Eds.), JFAK: Essays Dedicated to Johan van Benthem on the Occasion of his 50th Birthday (Vol. 3, pp. 25-36). Amsterdam: Amsterdam University Press.

Panisson, A. R., Sarkadi, S., McBurney, P., Parsons, S., and Bordini, R. H. (2018). On the Formal Semantics of Theory of Mind in Agent Communication, In 6th International Conference Agreement Technologies, AT 2018, Bergen, Norway, December 6-7, 2018, Revised Selected Papers, 18-32. https://nms.kcl.ac.uk/ simon.parsons/publications/conferences/at18a.pdf

Reed, C. and Norman, T. J., eds. (2004). Argumentation Machines: New Frontiers in Argument and Computation, Berlin: Springer.

Van Eemeren F. H. and Grootendorst, R. (2004), A Systematic Theory of Argumentation: the Pragma-dialectical Approach. Cambridge: Cambridge University Press.

Walton, D. and Krabbe, E. C. W. (1995). Commitment in Dialogue: Basic Concepts of Interpersonal Reasoning. Albany: SUNY University Press, 1995.

Walton, D., Reed, C. and Macago, F. (2008). Argumentation Schemes. Cambridge: Cambridge University Press, 2008.

9 See his Google Scholar page: https://scholar.google.com/citations?user=Zq2Ot4IAAAAJ\&hl=en 
Walton, D. and Gordon, T. F. Cumulative Arguments in Artificial Intelligence and Informal Logic, Revista Iberoamericana de Argumentacion, 14(2), 2017, 1-28. http://www.dougwalton.ca/papers\%20in\%20 pdf/17Revista.pdf

Walton, D., Toniolo, A. and Norman, T. J. (2019), Dialectical Models of Deliberation, Problem Solving and Decision Making, Argumentation, to appear: http://www.dougwalton.ca/papers\%20in\%20 pdf/19DelibWTN.pdf 